\title{
COCOMMUTATIVE HOPF ALGEBRAS WITH ANTIPODE
}

\author{
BY MOSS E. SWEEDLER ${ }^{1}$
}

Communicated by A. Mattuck, June 1, 1966

We shall describe the structure of a certain kind of Hopf algebra over an algebraically closed field $k$ of characteristic $p$, namely those Hopf algebras whose coalgebra structure is commutative and which have an antipodal map $S: H \rightarrow H$. (See below for definitions.) Such a Hopf algebra turns out to be of the form $k G \# U$, the smash product of a group algebra with a Hopf algebra whose coalgebra structure is "like" that of a universal enveloping algebra. If $p=0$ the second factor actually is a universal enveloping algebra.

For $p>0$, we generalize the Birkhoff-Witt theorem by introducing the notion of divided powers. These also play a role in the theory of algebraic groups where certain sequences of divided powers correspond to one parameter subgroups. The divided powers appear in a "Galois Theory" for all finite normal field extensions.

The structure theory of $Z_{2}$-graded coanticommutative Hopf algebras is similar, and mentioned below.

Lemma 1, Theorem 1, its generalization to the graded case, and Theorem 2 are unpublished results of $\mathrm{B}$. Kostant, whose guidance we gratefully acknowledge.

1. $H$ is a cocommutative Hopf algebra with multiplication $m$, augmentation $\epsilon$ and diagonal $d$.

Definition. An element $g \in H$ is grouplike if $d g=g \otimes g$ and $g \neq 0$.

Lemma 1. The set $G$ of grouplike elements of $H$ form a multiplicative semigroup whose elements are linearly independent in $H$. For each $g \in G$ there exists a unique maximal coalgebra $H^{\circ} \mathrm{CH}$ whose only grouplike element is g. $H \cong \oplus H^{g}$ as a coalgebra, and $H^{o} H^{h} \subset H^{o h}$.

Definition. $S: H \rightarrow H$ is an antipode if

$$
m \circ(I \otimes S) \circ d=\epsilon=m \circ(S \otimes I) \circ d .
$$

THEOREM 1. If $H$ has an antipode $G$ is a group and $S(g)=g^{-1}$. If $e$ is the identity of $G, H^{o}=g H^{e}=H^{e} g$, and $H \cong k G \# H^{e}$ as a Hopf algebra.

REMARK. Since $g^{-1} H^{e} g=H^{e}$, the elements of $G$ act as Hopf algebra automorphisms of $H^{e}$ and so we can form the smash product $k G \# H^{e}$.

${ }^{1}$ Part of the research described here was done while the author held an N.S.F. Graduate Fellowship. 
(As a coalgebra this is $k G \otimes H^{e},(1 \otimes h)(g \otimes 1)=\left(g \otimes g^{-1} h g\right) g \in G$, $h \in H^{e}$.)

If $F$ is a cocommutative Hopf algebra with one grouplike element, $G$ a group of Hopf algebra automorphisms of $F$ then $k G \# F$ has a unique antipode.

In the $Z_{2}$-graded coanticommutative situation, $G \subset H_{0}, H^{\circ}$ $=\left(H^{e} \cap H_{0}\right) \oplus\left(H^{e} \cap H_{1}\right)$. If $H$ has an antipode, $G$ is a group and $H \cong k G \# H^{e}$ as a graded Hopf algebra.

2. We now determine the structure of $H^{e}$, i.e. we consider a Hopf algebra $H$ with one grouplike element.

Theorem 2. If $p=0, H$ is the universal enveloping algebra of the Lie algebra $L$ (under $[]$,$) , where$

$$
L=\{x \in H \mid d x=x \otimes 1+1 \otimes x\} .
$$

Definition. For arbitrary $p$ the elements of $L$ are called primitive. If $p>0, L$ is a restricted Lie algebra but $H$ is not necessarily its restricted universal enveloping algebra. However, using the BirkhoffWitt theorem we can get a form of Theorem 2 which does generalize to $p>0$. Namely it says for $p=0, H=\otimes C_{\gamma}$ as a coalgebra, where $C_{\gamma}$ is the subspace of $H$ spanned by the elements ${ }^{e} l_{\gamma}=l_{\gamma}^{e} / e ! e=0,1, \ldots$ and $\left\{l_{\gamma}\right\}$ is a basis for $L$. Note that $C_{\gamma}$ is a coalgebra because $d^{e} l_{\gamma}$ $=\sum_{0}^{e}{ }^{i} l_{\gamma} \otimes{ }^{e-i} l_{\gamma}$.

Definition. A finite or infinite sequence of elements $1=0,{ }^{1} l$, ${ }^{2} l, \ldots$ is called a sequence of divided powers of ${ }^{1} l$ if $d^{n} l=\sum_{0}^{n}{ }^{i} l \otimes^{n-i} l$.

Given an indeterminate $x$, let $H_{x}^{\infty}$ be the Hopf algebra with a basis of indeterminates ${ }^{i} x, i=0,1,2, \cdots$, the algebra structure is determined by ${ }^{i} x^{i} x=\left(\begin{array}{c}i+j \\ j\end{array}\right) x^{i+j}$ and the coalgebra structure is determined by ${ }^{0} x,{ }^{1} x, \cdots$, which is a sequence of divided powers of ${ }^{1} x$. If $p>0$ we let $H_{x}^{n}$ be the sub-Hopf algebra spanned by ${ }^{0} x,{ }^{1} x, \cdots, p^{n-1} x$.

Let $H^{\prime}=\operatorname{Hom}(H, k)$ have the algebra structure "transpose" to the coalgebra structure of $H$. Thus for $a^{\prime}, b^{\prime} \in H^{\prime}, a^{\prime} * b^{\prime}$ is the map $\left(a^{\prime} \otimes b^{\prime}\right) \circ d: H \rightarrow k . H^{\prime}$ is a commutative algebra since $H$ is cocommutative.

TheOREM 3. For $p>0$, let $I^{n} \subset H^{\prime}$ be the ideal generated by $\left\{a^{\prime} \in H^{\prime} \mid a^{\prime} p^{n}=0\right\}$. If the sequence of ideals $I^{1} \subset I^{2} \subset \cdots$ terminates, then $H \cong \otimes H_{x}^{n_{x}}$ as a coalgebra, for some set of elements $\{x\}$ and positive integers $($ or $\infty),\left\{n_{x}\right\}$.

If $I^{1}=0, H \cong \otimes H_{x}^{\infty}$ as a coalgebra, where we may choose $\{x\}$ to be a basis for $L$. 
If $I^{1}=\left\{a^{\prime} \in H^{\prime} \mid a^{\prime}(1)=0\right\}$, then $H$ is the restricted universal enveloping algebra of $L$. So $H \cong \otimes H_{x}^{\prime}$ as a coalgebra, where $\{x\}$ is a basis for $L$.

The techniques involved in proving Theorem 3 yield information about sequences of divided powers lying above an element of $L$. For example, $l \in L$ is orthogonal to $I^{n}$ if and only if $l$ lies in a sequence of divided powers $0,{ }^{1} l=l,{ }^{2} l, \cdots p^{n+1}-1 l$.

In the coanticommutative situation the Hopf algebra $H$ contains a unique maximal sub Hopf algebra $F \subset H_{0}$. Theorem 2 or 3 applies to $F$. If $L_{0}=L \cap H_{0}$ and $L_{1}=L \cap H_{1}$ then $L=L_{0} \oplus L_{1}$ and $L$ is a graded Lie algebra. If $\Lambda L_{1}$ is the exterior algebra on $L_{1}$ then $H \cong F \otimes \Lambda L_{1}$ as a coalgebra. If $p=0, H$ is the graded universal enveloping algebra of $L$.

\section{BIBLIOGRAPHY}

1. E. Halpern, Twisted polynomial hyperalgebras, Mem. Amer. Math. Soc. No. 29, 1958.

2. J. W. Milnor and J. C. Moore, On the structure of Hopf algebras, Ann. of Math. (2) 81 (1965), 211-264.

Massachusetts Institute of Technology 